\title{
Heartbeat: Heart failure and obstructive lung disease: are beta-blockers underused?
}

Current guidelines for management of heart failure (HF) recommend beta-blocker therapy even in patients with chronic obstructive pulmonary disease (COPD). However, many clinicians find it counterintuitive to treat COPD patients with betablockers either because of concerns that beta-blockers might negate the effects of inhaled beta-agonists or because betablockers might even provoke bronchoconstriction. Lipworth and colleagues (see page 1909) studied the "real-life" use of betablocker therapy based on anonymous longitudinal electronic medical record data from over 2.4 million patients in the UK that included almost 90 thousand COPD and 24 thousand HF patients who were followed for more than one year. About 12\% of the COPD patients also had HF; these 10 thousand COPD +HF patients (mean age 79 years, $60 \%$ male) were compared to a similar number of patients with $\mathrm{HF}$ alone. In patients treated with an angiotensin converting enzyme inhibitor (ACEI) or angiotensin-2 receptor blocker (ARB), only $22 \%$ of the COPD patients also were taking a beta-blocker, compared to $41 \%$ of the patients with HF but no COPD (adjusted OR $0.54,95 \%$ CI 0.51 to $0.58, \mathrm{p}<0.001$ ) (figure 1). Similarly, beta blocker use was less common in COPD patients treated with inhaled medications.

This article generated a great deal of social media interest, primarily among members of the general public, rather than researchers or clinicians. In a letter to the editor, Cunnington (see page 1934) points out the importance of distinguishing patients with HF with preserved ejection fraction (HFpEF) from those with a reduced ejection fraction (HFrEF) because beta-blockers may not be beneficial for HFpEF and these patients may have significant comorbidities that may be difficult to take into account, even with this large database. In their reply, Lipworth and colleagues (see page 1934) respond that although they do not have precise data on HFpEF versus HFrEF, the prevalence of hypertension and diabetes was similar in those with $\mathrm{HF}$ alone compared to HF+COPD. They conclude "The higher use of ACEI/ARB

\footnotetext{
Correspondence to

Professor Catherine M Otto, Division of Cardiology, University of Washington, Seattle, WA 98195, USA; cmotto@u.washington.edu
}

without beta-blocker in HF with COPD compared to $\mathrm{HF}$ alone is more likely to reflect a reticence of physicians to prescribe add on therapy with beta-blockers in the presence of airflow obstruction due to fears of bronchoconstriction. As such we remain confident in the strength of our conclusions regarding underuse of beta-blockers in HF with COPD". Although initiating betablocker therapy in COPD patients can be challenging, observational data supports the mortality benefit of this therapy for $\mathrm{HF}$ even when COPD is present. Our goal should be ensure more patients with HF + COPD receive this therapy. Future studies should address the specific factors (clinical, genetic, pharmacologic) that affect the efficacy and tolerability of beta-blockers in these patients. However, a randomized clinical trial of HF treatment in COPD patients would provide the strongest evidence on which to base our clinical management.

Over the past 2 decades, there has been increasing evidence supporting antithrombotic therapy for prevention and treatment of cardiovascular disease, as well as the introduction of new antithrombotic medications. ${ }^{1}{ }^{2}$ Adelborg and colleagues (see page 1883) studied the long-term trends in use of anti-thrombotic agents in Denmark from 1999-2014 in order to better understand the transition from publication of new evidence to actual clinical decision making. Over this 16 year period, the prevalence of antithrombotic drug use increased from $5.1 \%$ to $9.6 \%$ of the Danish population with the rate of increase closely linked to publication of key clinical trials (figure 2). The impact of publication

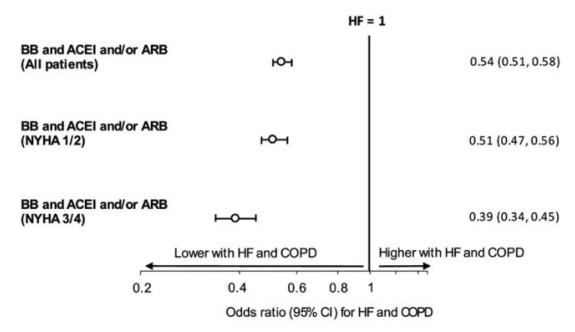

Figure 1 Prescription of $\beta$-blocker (BB) and ACEI (ACE inhibitor) or angiotensin-2 receptor blocker (ARB) for patients with heart failure (HF) alone versus patients with HF and chronic obstructive pulmonary disease (COPD)showing data for all patients and split by New York Heart Association (NYHA) class. Data for adjusted ORs are shown with $95 \% \mathrm{Cl}$. of new guideline documents was not included in this study.

In the accompanying editorial entitled "Antithrombotic drug use: scientific breakthroughs, biological limits, and physician behavior", Hylek (see page 1859) points out that despite the increased use of antithrombotic agents in Denmark, "the proportion of individuals with atrial fibrillation (AF) receiving no therapy or only aspirin for stroke prevention remains a troubling 30-40\%". She concludes: "Worldwide, thrombosis is responsible for one in four deaths. Because the risks for both haemorrhage and thrombosis increase with age, the search for interventions without attendant bleeding risk will continue. Innovations in materials science and engineering will hopefully create vascular device surfaces that are less thrombogenic. Long-term adherence to antithrombotic treatments remains a perpetual challenge, and the global disparities in access to proven therapies should haunt us all”.

The increasing prevalence of AF has led to further studies into early detection and treatment, particularly with respect to stroke prevention. ${ }^{3}{ }^{4}$ In a comparative study of AF patients enrolled in registries in the UK and Japan, Senoo and colleagues (see page 1878) examined clinical characteristics, stroke risk and outcomes to determine if geographic or ethnic differences were present in these populations. The overall 12 -month risk of stroke was $3.4 \%$ in Japan and $4.4 \%$ in the UK with similar baseline characteristics (mean age 82-84 years). Mortality at one year also was similar in both countries: $11.5 \%$ in Japan and $14.1 \%$ in the UK. In both registries, less than $60 \%$ of AF patients were receiving appropriate anti-thrombotic therapy. The multivariable analysis of risk factors associated with adverse outcomes in those not taking oral anticoagulation (table 1) showed no differences related to ethnicity, with the strongest risk factors being a history of stroke, older age, and vascular disease.

In an editorial, Ayabe and Goto (see page 1857) comment that "Most clinical hypotheses regarding treatment of cardiovascular diseases have been tested in clinical trials which assume homogeneity of patients around the world". "In reality, however, 'global trials' primarily have been conducted by recruiting patients from only selected regions of the world, especially 


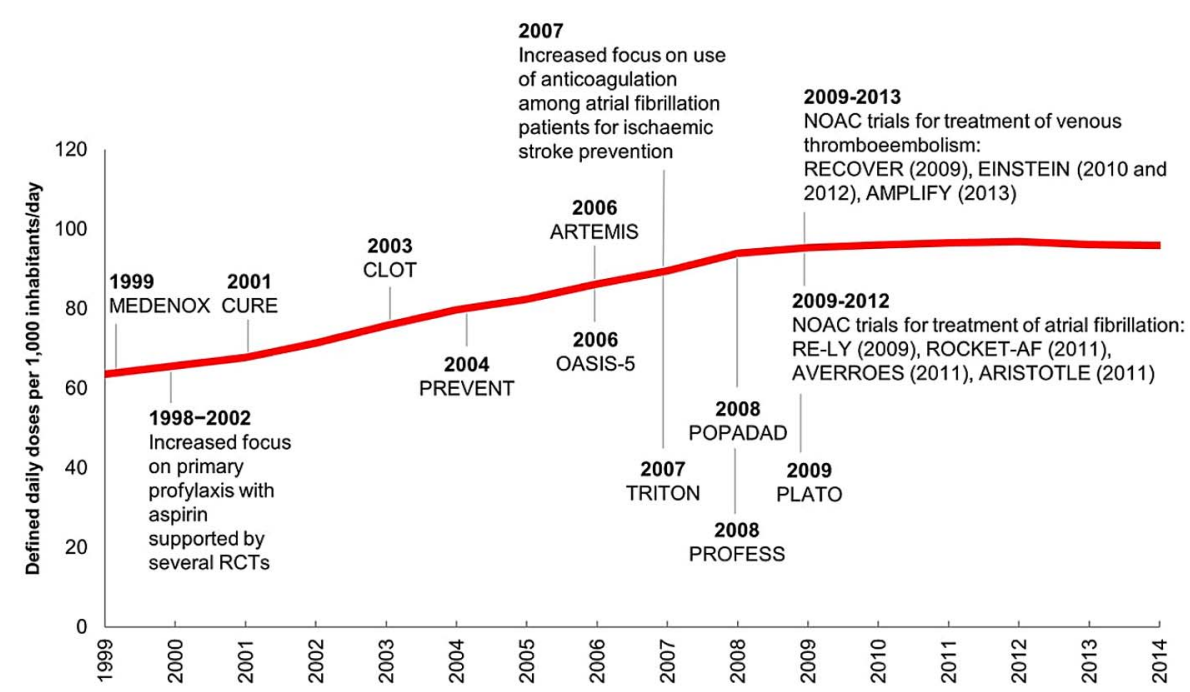

Figure 2 Timeline in use of antithrombotic drugs in Denmark and publication of landmark studies. AMPLIFY, oral apixaban for the treatment of acute venous thromboembolism study; ARISTOTLE, apixaban versus warfarin in patients with atrial fibrillation study; ARTEMIS, efficacy and safety of fondaparinux for the prevention of venous thromboembolism in older acute medical patients: randomised placebo-controlled trial; AVERROES, apixaban in patients with atrial fibrillation study; CLOT, low-molecular weight heparin versus a coumarin for the prevention of recurrent venous thromboembolism in patients with cancer study; CURE, effects of clopidogrel in addition to aspirin in patients with acute coronary syndromes without ST-segment elevation study; EINSTEIN, oral rivaroxaban for symptomatic venous thromboembolism study; MEDENOX, a comparison of enoxaparin with placebo for the prevention of venous thromboembolism in acutely ill medical patients study; NOAC, non-vitamin K oral antagonists; OASIS 5, comparison of fondaparinux and enoxaparin in acute coronary syndromes study; PLATO, ticagrelor versus clopidogrel in patients with acute coronary syndromes study; POPADAD, the prevention of progression of arterial disease and diabetes trial; PREVENT, randomised, placebo-controlled trial of dalteparin for the prevention of venous thromboembolism in acutely ill medical patients study; PROFESS, aspirin and extended-release dipyridamole versus clopidogrel for recurrent stroke study; $\mathrm{RCT}$, randomised controlled trial; RECOVER, dabigatran versus warfarin in the treatment of acute venous thromboembolism study; RE-LY, dabigatran versus warfarin in patients with atrial fibrillation study; ROCKET-AF, rivaroxaban versus warfarin in non-valvular atrial fibrillation study; TRITON-TIMI 38, prasugrel versus clopidogrel in patients with acute coronary syndromes study.

Table 1 Multivariable logistic regression analysis for stroke and death in patients not receiving oral anticoagulation ( $n=1489$; Japan from Fushimi AF Registry $(n=798)$ and the UK from Darlington AF Registry $(n=691))$

\begin{tabular}{llllc}
\hline & $\begin{array}{l}\text { Stroke } \\
\text { OR }(95 \% \mathrm{Cl})\end{array}$ & p Value & $\begin{array}{l}\text { Death } \\
\text { OR }(95 \% \mathrm{Cl})\end{array}$ & p Value \\
\hline Age & $0.99(0.95$ to 1.03$)$ & 0.68 & $1.10(1.07$ to 1.13$)$ & $<0.001$ \\
Female & $1.59(0.93$ to 2.79$)$ & 0.09 & $1.10(0.81$ to 1.49$)$ & 0.54 \\
Heart failure & $1.04(0.57$ to 1.83$)$ & 0.89 & $1.64(1.20$ to 2.24$)$ & 0.002 \\
Hypertension & $1.53(0.87$ to 2.84$)$ & 0.14 & $0.81(0.60$ to 1.10$)$ & 0.18 \\
Diabetes mellitus & $1.47(0.82$ to 2.53$)$ & 0.19 & $1.17(0.81$ to 1.65$)$ & 0.40 \\
Previous stroke & $2.42(1.39$ to 4.12$)$ & 0.002 & $1.62(1.14$ to 2.27$)$ & 0.01 \\
Vascular disease & $1.29(0.65$ to 2.39$)$ & 0.45 & $2.36(1.64$ to 3.37$)$ & $<0.001$ \\
Use of antiplatelet agents & $1.20(0.68$ to 2.15$)$ & 0.54 & $0.39(0.28$ to 0.54$)$ & $<0.001$ \\
Ethnicity * (Japan vs UK) & $0.86(0.50$ to 1.47$)$ & 0.58 & $0.67(0.49$ to 0.92$)$ & 0.01 \\
\hline *UK as a reference. & & & & \\
AF, atrial fibrillation & & & &
\end{tabular}

Europe and North America. Currently, many Asian countries are starting to participate in major clinical trials, but we should recognise that most did not include substantial number of Asian patients until quite recently". "Readers will be interested to

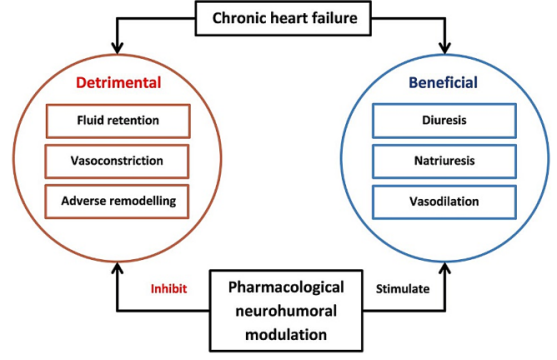

Figure 3 Contrasting effects of neurohumoral modulation. Neurohumoral activation of renin-angiotensin-aldosterone system and sympathetic nervous system in chronic heart failure cause several adverse effect including (1) fluid retention, (2) peripheral vasoconstriction and (3) adverse cardiac remodelling. These detrimental responses predominate in chronic heart failure and current pharmacological treatment has focused on inhibiting these effects. Potentially beneficial, but weaker, vasodilatory and natriuretic pathways are also activated in chronic heart failure. Future progress in our ability to assess the individual neurohumoral systems could allow for therapy tailored to the individual patient's status. This opens the possibility for a more comprehensive pharmacological neuromodulation.

best 'standard of care' at the east end of the Eurasian continent should differ from the west end or not".

The Education in Heart article in this issue (see page 1922) discusses the pathophysiologic basis and clinical implementation of optimal neuro-humoral antagonism in patients with chronic heart failure (figure 3).

Check out the Image Challenge (see page 1889); it shows a common, but often misinterpreted, echocardiographic finding.

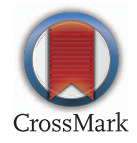

To cite Otto CM. Heart 2016;102:1855-1856.

Heart 2016;102:1855-1856.

doi:10.1136/heartjnl-2016-310817

\section{REFERENCES}

1 Dogliotti A, Paolasso E, Giugliano RP. Current and new oral antithrombotics in non-valvular atrial fibrillation: a network meta-analysis of 79808 patients. Heart 2014;100:396-405.

2 De Caterina R, Ammentorp B, Darius H, et al. PREFER in AF Registry Investigators. Frequent and possibly inappropriate use of combination therapy with an oral anticoagulant and antiplatelet agents in patients with atrial fibrillation in Europe. Heart 2014;100:1625-35.

realise that there is a difference in the 'standard of care' for anticoagulant therapy in elderly patients with $\mathrm{AF}$ who live on these two islands located at the extreme east and west ends of the Eurasian continent". "It remains uncertain whether the
3 Martinez C, Katholing A, Wallenhorst C, et al. Increasing incidence of non-valvular atrial fibrillation in the UK from 2001 to 2013. Heart 2015;101:1748-54.

4 Keach JW, Bradley SM, Turakhia MP, et al. Early detection of occult atrial fibrillation and stroke prevention. Heart 2015;101:1097-102. 